\title{
Supplement to manuscript: Bayesian inverse modeling and source location of an unintended I-131 release in Europe in the fall of 2011
}

Ondřej Tichý ${ }^{1}$, Václav Šmídl ${ }^{1}$, Radek Hofman ${ }^{1}$, Kateřina Šindelářová ${ }^{1}$, Miroslav Hýža ${ }^{2}$, and Andreas Stohl ${ }^{3}$

${ }^{1}$ Institute of Information Theory and Automation, Czech Academy of Sciences, Prague, Czech Republic

${ }^{2}$ National Radiation Protection Institute, Prague, Czech Republic

${ }^{3}$ NILU: Norwegian Institute for Air Research, Kjeller, Norway

Correspondence to: Ondřej Tichý (otichy@utia.cas.cz)

\section{Parameters for model selection}

The moments of the respected distributions are denoted with the hat symbol, $\widehat{\theta}$.

The terms related to $\mathrm{E}\left[\ln p\left(\mathbf{y}, \mathbf{x}, \Upsilon, L, \boldsymbol{\psi}, \omega, M_{i}\right)\right]$ are:

$$
\ln p(\mathbf{y} \mid \mathbf{x}, \omega)=-\frac{p}{2} \ln (2 \pi)+\frac{p}{2} \widehat{\ln \omega}-\frac{1}{2} \widehat{\omega}\left(\operatorname{tr}\left(\widehat{\mathbf{x x}^{T}} M^{T} M\right)-2 \mathbf{y}^{T} M \widehat{\mathbf{x}}+\mathbf{y}^{T} \mathbf{y}\right),
$$

5

$$
\ln p(\omega)=-\ln \Gamma\left(\vartheta_{0}\right)+\vartheta_{0} \ln \rho_{0}+\left(\vartheta_{0}-1\right) \widehat{\ln \omega}-\rho_{0} \widehat{\omega},
$$

$$
\begin{aligned}
\ln p(\mathbf{x} \mid \boldsymbol{v}, L) & \left.=-\frac{n}{2} \ln (2 \pi)+\frac{1}{2} \widehat{\ln |\Upsilon|}-\frac{1}{2}\left(\mathbf{x}^{T} \widehat{\left(L \Upsilon L^{T}\right.}\right) \mathbf{x}\right) \\
& \left.=-\frac{n}{2} \ln (2 \pi)+\frac{1}{2} \sum_{j=1}^{n} \widehat{\ln v_{j}}-\frac{1}{2} \operatorname{tr}\left(\mathbf{x}^{T} \widehat{\left(L \Upsilon L^{T}\right.}\right) \mathbf{x}\right) \\
& =-\frac{n}{2} \ln (2 \pi)+\frac{1}{2} \sum_{j=1}^{n} \widehat{\ln v_{j}}-\frac{1}{2} \operatorname{tr}\left(L^{L^{T} \mathbf{x x}^{T}} L \widehat{\Upsilon}\right)
\end{aligned}
$$

$$
\ln p\left(x_{j} \mid \boldsymbol{v}, L\right)=\ln \sqrt{2}-\frac{1}{2 \sigma_{x_{j}}} \widehat{x_{j}^{2}}-\ln \sqrt{\pi \sigma_{x_{j}}}-\ln \left(1-\operatorname{erf}\left(\frac{0}{\sqrt{2 \sigma_{x_{j}}}}\right)\right)
$$

$$
\begin{gathered}
\sigma_{x_{j}} \rightarrow\left(\left(\widehat{L \Upsilon L^{T}}\right)^{-1}\right)_{j, j} \\
\ln p(\boldsymbol{v})=-n \ln \Gamma\left(\alpha_{0}\right)+n \alpha_{0} \ln \beta_{0}+\sum_{j=1}^{n}\left(\alpha_{0}-1\right) \widehat{\ln v_{j}}-\sum_{j=1}^{n} \beta_{0} \widehat{v_{j}}, \\
\left.\left.\left.\ln p\left(\mathbf{l}_{j} \mid \boldsymbol{\varsigma}_{j}\right)=-\frac{r s}{2} \ln (2 \pi)+\sum_{m=1}^{r s} \frac{1}{2} \widehat{\ln \varsigma_{j, m}}-\frac{1}{2}\left(\mathbf{l}_{j}^{T} \widehat{\operatorname{diag}\left(\boldsymbol{\varsigma}_{j}\right.}\right) \mathbf{l}_{j}-2 \mathbf{l}_{0, j}^{T} \widehat{\operatorname{diag}\left(\boldsymbol{\varsigma}_{j}\right.}\right) \widehat{\mathbf{l}_{j}}+\mathbf{l}_{0, j}^{T} \widehat{\operatorname{diag}\left(\boldsymbol{\varsigma}_{j}\right.}\right) \mathbf{l}_{0, j}\right), \\
\ln p\left(\boldsymbol{\varsigma}_{j}\right)=-r s \ln \Gamma\left(\zeta_{0}\right)+r s \zeta_{0} \ln \eta_{0}+\sum_{m=1}^{r s}\left(\zeta_{0}-1\right) \widehat{\ln \varsigma_{j, m}}-\sum_{m=1}^{r s} \eta_{0} \widehat{\varsigma_{j, m}} .
\end{gathered}
$$


The terms related to $\mathrm{E}[\ln \tilde{p}(\omega)]-\mathrm{E}[\ln \tilde{p}(\mathbf{x})]-\mathrm{E}[\ln \tilde{p}(\Upsilon)]-\mathrm{E}[\ln \tilde{p}(L)]-\mathrm{E}[\ln \tilde{p}(\boldsymbol{\psi})]$ are:

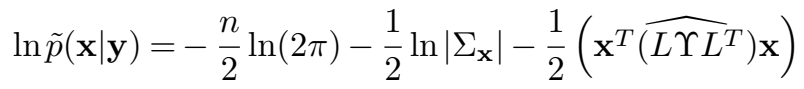

$$
\begin{aligned}
& \ln \tilde{p}\left(x_{j} \mid \mathbf{y}\right)=\ln \sqrt{2}-\frac{1}{2 \sigma_{x_{j}}}\left(\widehat{x_{j}}-\mu_{j}\right)^{2}-\ln \sqrt{\pi \sigma_{x_{j}}}-\ln \left(1-\operatorname{erf}\left(\frac{0-\mu_{x_{j}}}{\sqrt{2 \sigma_{x_{j}}}}\right)\right) \\
& \ln \tilde{p}(\boldsymbol{v} \mid \mathbf{y})=-\sum_{j=1}^{n} \ln \Gamma\left(\alpha_{j}\right)+\sum_{j=1}^{n} \alpha_{j} \ln \beta_{j}+\sum_{j=1}^{n}\left(\alpha_{j}-1\right) \widehat{\ln v_{j}}-\sum_{j=1}^{n} \beta_{j}{\widehat{v_{j}}} \\
& 5 \quad \ln \tilde{p}\left(\mathbf{l}_{j} \mid \mathbf{y}\right)=-\frac{r s}{2} \ln (2 \pi)-\frac{1}{2} \ln \left|\Sigma_{\mathbf{l}_{j}}\right|-\frac{1}{2}\left(\left(\widehat{\mathbf{l}_{j}}-\mu_{\mathbf{l}_{j}}\right)^{T} \Sigma_{\mathbf{l}_{j}}^{-1}\left(\widehat{\mathbf{l}_{j}}-\mu_{\mathbf{l}_{j}}\right)\right) \\
& \ln \tilde{p}\left(\boldsymbol{\varsigma}_{j} \mid \mathbf{y}\right)=-\sum_{m=1}^{r s} \ln \Gamma\left(\zeta_{m}\right)+\sum_{m=1}^{r s} \zeta_{m} \ln \eta_{m}+\sum_{m=1}^{r s}\left(\zeta_{m}-1\right) \widehat{\ln \varsigma_{j, m}}-\sum_{m=1}^{r s} \widehat{\eta_{m}} \widehat{\varsigma_{j, m}}, \\
& \ln \tilde{p}(\omega \mid \mathbf{y})=-\ln \Gamma(\vartheta)+\vartheta \ln \rho+(\vartheta-1) \widehat{\ln \omega}-\rho \widehat{\omega} .
\end{aligned}
$$

